\title{
Airway obstruction following palatoplasty of a patient with sleep disturbance
}

\author{
Ki-Bum Park, Hyun-Jung Lee, Hoon Jung, Seong Wook Hong, and Kyung-Hwa Kwak \\ Department of Anesthesiology and Pain Medicine, Kyungpook National University School of Medicine, Daegu, Korea
}

Cleft palate is the most common craniofacial anomaly, with an incidence of 1 in 700 live births. Primary corrective palatal surgery is often performed between 6 and 12 months. Although cleft palate closure may result in upper airway obstruction, tracheostomy is a rare complication after repair of cleft palate in patients without any other craniofacial abnormalities [1]. Here, we report a child who was presented without other craniofacial abnormalities but required unplanned tracheostomy to resolve an airway compromised after repair of cleft palate.

A 9-month old female weighing $7.4 \mathrm{~kg}$ was scheduled for correction of complete cleft palate. History taking from her parents revealed that the baby had difficulty in breathing in the supine position but appeared to breathe comfortably in the left or right lateral decubitus position during sleep. Her chest radiograph did not show any abnormal findings. Preoperative 3D-computed tomography (CT) of the head and neck revealed sufficient airway patency (Fig. 1A). Other laboratory findings were within normal ranges. In the operating room, the initial oxygen saturation was $99 \%$ in room air, and other vital signs were stable. We induced sevoflurane in gradual increments from 2 to $4 \%$ with oxygen using a mask and injected rocuronium bromide $3 \mathrm{mg}$. We intubated the patient with armored tube $3.0 \mathrm{~mm}$ at $11 \mathrm{~cm}$ from the incisor. Laryngoscopy visual field according to the Cormack-Lehane grades was grade 2. General anesthesia was maintained with sevoflurane (end-tidal concentration of 1.0-2.0\%). Operation time was about fifty minutes. At the end of surgery, the operator notified us that the posterior part of the tongue was significantly large. Extubation was carried out when
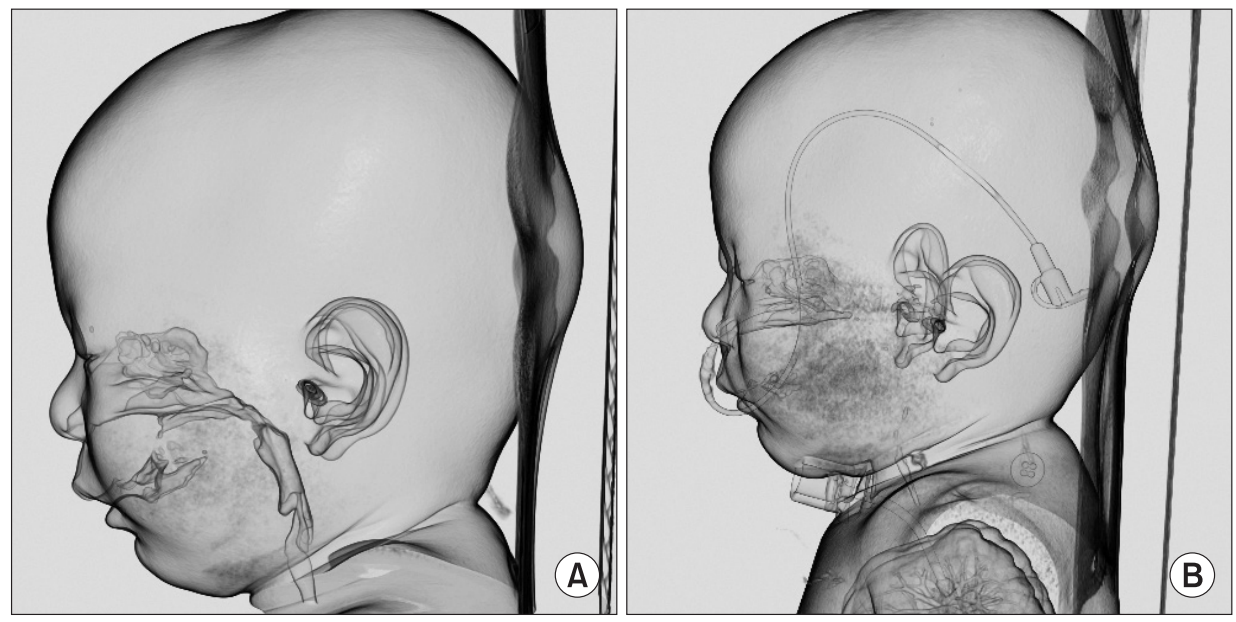

Fig. 1. (A) Preoperative computed tomography reveals sufficient airway patency. (B) Postoperative computed tomography taken 7 days after surgery shows no upper airway patency.

Corresponding author: Kyung-Hwa Kwak, M.D., Department of Anesthesiology and Pain Medicine, Kyungpook National University School of Medicine, 680, Gukchabosang-ro, Jung-gu, Daegu 700-842, Korea. Tel: 82-53-200-8788, Fax: 82-53-426-2760, E-mail: kwakkh@knu.ac.kr (c) This is an open-access article distributed under the terms of the Creative Commons Attribution Non-Commercial License (http:// creativecommons.org/licenses/by-nc/3.0/), which permits unrestricted non-commercial use, distribution, and reproduction in any medium, provided the original work is properly cited. 
the patient became completely alert and showed active spontaneous movement of the limbs. After extubation, desaturation started to get worse, and chest retraction was observed. At first, we presumed that desaturation was due to laryngospasm and attempted positive ventilation with a mask. However, airway obstruction did not improve and $\mathrm{SpO}_{2}$ dropped to $75 \%$ for 3 minutes. Reintubation was tried twice with a conventional tube 3 or a $3.5 \mathrm{~mm}$-sized tube, but failed. While elevating tongue with laryngoscope to intubate, chest retraction was resolved and $\mathrm{O}_{2}$ saturation reached up to $99 \%$. Thus, the surgeon pulled the tongue and tied it into the posterior wall of the lip to secure the airway. However, this was not effective enough to relieve the obstruction of the airway. Finally, we decided to carry out tracheostomy because the obstruction of the airway was not caused by oral or pharyngeal edema but by glossoptosis with the large posterior part of the tongue. An otolaryngologist performed tracheostomy with an uncuffed portex tracheostomy tube, $3.5 \mathrm{~mm}$.Upper airway obstruction by glossoptosis was observed in postoperative 3D-CT checked 7 days later (Fig. 1B). The patient was discharged without other complications 9 days after tracheostomy, with plans to reconstruct the tracheostomy site when the patient is old enough to secure airway patency.

Various factors other than craniofacial abnormalities affect upper airway patency after cleft palate repair. In addition, infants are obligate nasal breathers for the first few months of life, so they may be seriously affected by these factors. First, obstructive sleep apnea (OSA) is more common in the cleft population than the general population [2]. Second, simple closure as well as Furlow double opposing Z-plasty for lengthening and thicken- ing the palate or pharyngeal flap may increase the risk of airway obstruction [3,4]. Third, after repair of cleft palate, airway could be compromised by laryngospasm, blood clot, retained throat pack, or tongue swelling. In the present case, excessive blood clot, retained throat pack or tongue swelling was not observed. However, according to the patient's history from her parents, she had difficulty breathing in the supine position but appeared to breathe comfortably in the decubitus position during sleep. More precisely, this symptom suggests the potential existence of OSA, which might be due to the large posterior part of the tongue or glossoptosis during sleep. Postoperative CT (Fig. 1B) revealed that glossoptosis caused complete airway obstruction, which was suspected to be the main reason of airway disturbance. According to Smith et al. [5] post-operative respiratory distress after cleft palate repair is associated with OSA, not with orofacial syndromes such as Pierre Robin syndrome and Stickler syndrome. Therefore, preoperative sleep history or sleep study is important because reduced airway reserve in children with OSA may aggravate airway patency following cleft palate repair. Polysomnography can differentiate between central type and obstructive type of OSA. Sleep endoscopy and sleep fluoroscopy can be used to find the site of obstruction in the obstructive type of OSA.

In conclusion, repair of cleft palate may compromise airway patency of the patient in various ways during the postoperative period. The present case emphasizes the importance of detailed preoperative evaluation of risk factors suggestive of potential airway obstruction including sleep investigations in patients undergoing cleft palate repair.

\section{References}

1. Antony AK, Sloan GM. Airway obstruction following palatoplasty: analysis of 247 consecutive operations. Cleft Palate Craniofac J 2002; 39: 145-8.

2. Robison JG, Otteson TD. Increased prevalence of obstructive sleep apnea in patients with cleft palate. Arch Otolaryngol Head Neck Surg 2011; 137: 269-74.

3. Muntz H, Wilson M, Park A, Smith M, Grimmer JF. Sleep disordered breathing and obstructive sleep apnea in the cleft population. Laryngoscope 2008; 118: 348-53.

4. Liao YF, Chuang ML, Chen PK, Chen NH, Yun C, Huang CS. Incidence and severity of obstructive sleep apnea following pharyngeal flap surgery in patients with cleft palate. Cleft Palate Craniofac J 2002; 39: 312-6.

5. Smith D, Abdullah SE, Moores A, Wynne DM. Post-operative respiratory distress following primary cleft palate repair. J Laryngol Otol 2013; 127: 65-6. 